\title{
Generating 3D visual expression using semantic simplification description based on logical representation
}

\begin{abstract}
Performance on a question answering system is reflected by the number of correct answers that is produce from a list of alternate choices. However, many answers would appear in documents and passages laden with terms from the query. It becomes more difficult and eventually impossible to make a precise answer to a query. The aim of this study is to determine the keyword of the precise answer to a specific query and illustrate the answer in visual expression. In this paper we propose a method to translate text into visual expression, closest to the correct answer. To achieve a significant illustration capability it is necessary to develop methods of dealing with knowledge base. Firstly, we must consider the real world and the visual expression of key information that can be extracted from the words. The proposed system will analyse and match them using visual semantic simplification description based on logical representation.
\end{abstract}

Keyword: Visual expression; Precision answer extraction; Question answering system; Logical representation 